\title{
Computational Analysis on Electromagnetic Field Reversal based on Global Optimization Algorithm
}

\author{
Yanfeng Liu \\ College of Physics and Electronic Information, Yan'an University, Yanan, 716000, China
}

Keywords: Global optimization algorithm, Electromagnetic field, Reversal, Calculation

\begin{abstract}
With continuous development of computer technology and extensive research on electromagnetic field reversal, calculation of electromagnetic field has already become a research hotspot at present. Based on global optimization algorithm, the author discusses calculation of electromagnetic field reversal in this paper, with expectation to make an in-depth and comprehensive research on this question and make effective improvement to the calculation efficiency.
\end{abstract}

\section{Introduction}

Reversal of electromagnetic field is an important part of theoretical and numerical computation method of electromagnetic field. However, it is incomplete for calculation of its reversal at present. It lacks relatively mature support of theoretical system. More importantly, it also has no any complete and reliable algorithm ${ }^{[1]}$. Based on global optimization algorithm, therefore, the author discusses the calculation of electromagnetic field reversal in this paper.

\section{Overview of global optimization algorithm for calculation of electromagnetic field reversal}

\section{Basic concept of genetic algorithm}

Genetic algorithm is a set of computer model established with Darwinian biological evolution process as the template. From the 1960s to the present, this algorithm has already formed a set of extremely complete procedures. As genetic algorithm combined genetics and computer science, many genetic terms are involved in the establishment process of the model. Therefore, the author understood these terms, which is of great assistance to the understanding of genetic algorithm, for instance, chromosome, individual, population, gene, genotype, phenotype, fitness, encoding, decoding, choice, intersection and variation. In genetic algorithm, a certain number of individuals encoded by gene constitute the population of potential solution set of the question. In genetics, individual refers to those entities with chromosome features. As the major carrier of genetic material, however, chromosome is set of several genes. Therefore, external characters of individual are determined by internal gene combination. Thus, genetic development is used to realize encoding from phenotype to genotype and obtain the first generation of population, namely solution set of the first question. Then, appropriate individuals are selected according to the theory of survival of the fittest, and subject to intersected recombination of two chromosomes by virtue of genetic operator, or chromosome variation is generated to make a certain gene on chromosome to change, thus to represent new external characters and generate a new solution set. According to the theory of natural heredity, new descendants certainly will be more adaptive to the environment than previous generations. Therefore, the last population obtained at last can be the optimal solution set of the question ${ }^{[2]}$.

\section{Basic principle of particle swarm optimization}

Particle swarm optimization is a random search algorithm proposed by Kenney and Eberhart in 1995. This algorithm a population one evolved with reference to bird predation. At the very beginning, this algorithm makes one group of solutions to be random by means of initialization. Then, the optimal solution is found by successive iteration and renewal. This method is simple, and can be 
easily realized. Besides, it can also obtain good effect in such aspects as multi-target optimization, data classification, pattern recognition, signal processing, robot control and system identification. In particle swarm optimization, each solution which can optimize the question is treated as a tiny "particle". However, the fitness of these particles is determined by the optimized function; while the flight direction and distance of particle is determined by specific speed. Thus, particles are converted into the model of a flying bird. As a result, particle swarms will search in the space with the optimal particle, just the same as a foraging bird, so as to realize the purpose of coexistence and cooperative optimization by multiple particles. Each particle will combine relevant experiences of particle swarm and itself to fly toward a more perfect direction in the space, so as to search the optimal solution. Let's suppose the function controlling the flight direction and distance of particle as F, at first, initialize position vector and velocity vector of the particle; then, the particle will renew its flight direction and distance in the next step according to the optimal solution searched (extreme value of individual) and the optimal solution (global extreme value) found by the entire particle swarm. After such successive renewal and iteration, the particle will reach a velocity limit, so as to find the optimal solution ${ }^{[3]}$.

\section{Realization flow of global optimization algorithm for calculation of electromagnetic field reversal}

\section{Realization flow of genetic algorithm}

Basic operation of genetic algorithm mainly includes copy, intersection and variation. Copy mainly refers to the process in which individuals with the biggest resource superiority are selected to realize its copy and finish creation of new population according to individual features in the last generation of resources. Many algorithms are involved in copy operation. Among these algorithms, roulette algorithm is used the most frequently. At the same time, it is also the most classic one. Intersection is the most important part in genetic algorithm, which masters the effectiveness of genetic algorithm. In this algorithm, intersection algorithm is mainly completed in two parts: the first part is to randomly draw two individual resources among existing group resources and then take the two resources as parent individuals of interlace operation. The second part is to make random selection of intersections and realize intersected reproduction of matched bit string to generate new bit string. Mutation operation plays a crucial role in genetic algorithm. If genetic algorithm only includes copy and interlace operations, the obtained result will be similar to inbreeding. Due to finiteness of group resources and race resources, this race of kin and its resources will be eventually made to continuously expand through several interlace operations of kin. At the same time, resource superiority will be gradually decreased, making it fail to obtain the optimal solution. Therefore, to avoid occurrence of this phenomenon in genetic algorithm, genetic development in the living nature should be adopted to realize genovariation and perform small probability substitution for individual ${ }^{[4]}$. General flow is shown as follows:

Step 1: to realize the feasibility of genetic algorithm, initial population should be subject to random generation and selection. At the same time, the number of individuals should be quantitatively regulated, and these individuals should also be subject to chromogene encoding;

Step 2: fitness of population individual should be calculated according to genetic requirements. Based on the calculated result, judge whether it conforms to the optimal principle of heredity; if the selected individuals conform to the optimal principle, output it and end this operation on behalf of the optimal solution;

Step 3: in case of nonconformity, reselect individual resources till the optimal individual is selected; other individuals with relatively low fitness can be eliminated;

Step 4: realize generation of new individuals according to specific crossover probability and method;

Step 5: generate new individuals according to its variation principle;

Step 6: complete crossover and mutation operation, create a new population, and back to step 2 


\section{Realization flow of particle swarm optimization}

Particle swarm optimization belongs to a new evolutionary algorithm developed in recent years. In the implementation process, it is initialized into a group of random particles. Then, the optimal solution is found through iteration. In the process of iteration, particle may realize its renewal by the optimal solution found by itself or the entire population. Extreme value of particle is also extreme value of individual; while extreme value of population represents global extreme value. At the same time, it is also unnecessary to apply the entire population in the implementation process. Instead, only a part can be used as particle neighbor. Therefore, its peripheral extreme value is local extreme value. Specific implementation flow is shown as follows:

1). Initialize existing particles, set the number of particles as $\mathrm{N}_{\text {popsize, }}$ and calculate random initial solutions of set particles to generate the initial speed of $\mathrm{N}_{\text {popsize; }}$;

2). Renew location and velocity vectors of particles at present according to their locations and velocity;

3). After realization of above operation, calculate adaptive value of new positions of existing particles in the particle swarm; if the particle adaptive value at the new position is of greater superiority than individual extreme value of the original particle $\mathrm{N}_{\text {best }}$, renew individual extreme value;

4). Renew global extreme value $\mathrm{G}_{\text {best }}$ according to new extreme value of existing particles;

5). Realize particle velocity renewal according to step (1);

6). Realize location renewal according to step (3);

7). Quit if meeting end conditions (the maximum number of circulation equivalent to error); or, back to step (3) .

\section{Advantages and disadvantages of global optimization algorithm for calculation of electromagnetic field reversal}

\section{Advantages and disadvantages of genetic algorithm}

\section{Advantages}

1). Genetic algorithm is of universality for expression of feasible solutions. Its processing object includes some gene individuals obtained after encoding with parameter set instead of parameters themselves. Therefore, it has certain superiority to traditional algorithms. In this process, encoding leaves out some surplus procedures, and structural objects can be directly operated. This is also an important feature for wide application of genetic algorithm [5].

2). Genetic algorithm has the feature of group search. It has better search performance than traditional algorithms. The operational method of simultaneous processing of multiple individuals in the group is adopted in genetic algorithm. It realizes comprehensive assessment for multiple solutions within the search spatial range at the same time. However, search method is simple in traditional algorithms, namely point-to-point search. In complicated search, it is easy for traditional algorithms to be got into local extreme value, and difficult to finish comprehensive search and assessment. Therefore, genetic algorithm can be used to good global search when compared with traditional algorithms.

3). It is unnecessary for genetic algorithm to have relevant supplementary information. In genetic algorithm, gene individuals are subject to comprehensive assessment through the numerical value of fitness function. Relevant genetic operations are performed by this means. Genetic algorithm is provided with a wide range of fitness function. Compared with traditional algorithms, genetic algorithm is not limited by continuously differentiable conditions. At the same time, domains of definition of these fitness functions can be randomly set. Genetic algorithm has a relatively wide range of application. More importantly, it has less restricted conditions. However, encoding of fitness function needs to be corresponding to the feasible solution space, so as to avoid the occurrence of dead code. 
4). Genetic algorithm has the function of inherent heuristic random search. Its search has global features. For its application, the transition rule of probability is adopted to determine relevant search directions. It is unnecessary for definite rules of traditional algorithms. Genetic algorithm has the function of inherent heuristic random search. Each search individual can be assessed in relevant groups, so as to obtain the best gene individuals, finish effective search, and improve the searching efficiency on this basis. At the same time, application of genetic algorithm is expandable. In relevant operations, it can be effectively combined with other techniques.

5). Genetic algorithm can realize finding of the optimal solution. Genetic algorithm is provided with strong parallel computing power. In the search process, it can realize global search processing. Compared with traditional algorithms, it can find the optimal solution within the global range to a large extent. Natural evolution mechanism is adopted in genetic algorithm, which can quickly and effectively process relatively complex questions.

\section{Disadvantages}

Genetic algorithm optimized and improved traditional algorithms. Therefore, it still has some disadvantages, for instance:

1). Genetic algorithm has standardized requirements for encoding. At the same time, it is also inaccurate for expression to existence of encoding.

2). For relatively simple genetic codes, genetic algorithm will increase the calculation time. It is necessary to adopt threshold value for non-feasible solution.

3). Compared with traditional algorithms, genetic algorithm has a more complicated process. Therefore, its efficiency is lower than traditional optimization methods.

4). It requires a certain calculation process in traditional algorithms. However, genetic algorithm is of great randomness. Therefore, it is easy for premature convergence.

5). Genetic algorithm can realize effective search, but there is no definite quantitative analysis method for its precision, complexity and reliability.

\section{Advantages and disadvantages of particle swarm optimization}

\section{Advantages}

Particle swarm optimization has the following advantages: it can be used to process some questions that cannot be solved with traditional algorithms. For instance, particle swarm optimization has better processing effect for relevant questions existing in non-derivable node function and without relevant gradient information. Advantages of particle swarm optimization can be analyzed with reference to features of genetic algorithm. Relevant advantages are shown as follows:

1). Both algorithms belong to the category of bionic algorithm. Particle swarm optimization is a kind of evolutionary computation method developed from the research on bird predation, human cognition and some relevant social behaviors. Genetic algorithm was proposed mainly by applying and developing the theory of survival of the fittest in the nature.

2). Both algorithms belong to the category of global optimization. Both particle swarm optimization and genetic algorithm can be used to search in the solution space within the global range. Moreover, the search result of both algorithms can realize finding of the optimal solution. At the same time, it is of greater search importance when compared with traditional methods.

3). Both algorithms belong to random search methods. In the search process of particle swarm optimization, system initialization is a group of random solutions. The optimal solution is searched through iteration. In genetic algorithm, the optimal solution is continuously searched by random operation in a certain group. Genetic operation belongs to random operation.

4). Both algorithms have the feature of parallelism. Parallelism is an important precondition for wide application of an algorithm. In both particle swarm optimization and genetic algorithm, search begins from a corresponding set. The search process has the feature of parallelism, which increases the search probability.

5). Both algorithms are hardly limited. They both begin to search according to the fitness information of individual. Therefore, neither particle swarm optimization nor genetic algorithm is restrained by function condition. 


\section{Disadvantages}

1). Particle swarm optimization has disadvantage for processing of high-dimensional and complex questions. Its operation process is of great randomness, and it is easy for premature convergence for processing of high-dimensional and complex questions.

2). Particle swarm optimization represents a kind of single information sharing. Therefore, its operation is of certain limitation.

3). Particle swarm optimization has no crossover or mutation operation. It is only of internal renewal and search, with poor global grasp.

4). Particle swarm optimization is of a narrow application range. It is mainly applied in solving some continuous questions. However, it cannot be used to solve discretized questions.

\section{Conclusion}

Reversal of electromagnetic field mainly refers to achievement of given ideal performance indexes and parameters of an electromagnetic device by optimization of this device. Based on global optimization algorithm, the author discusses calculation of electromagnetic field reversal, which can effectively reduce the number of calculation in electromagnetic devices, and guarantee the obtainment of the optimal solution with this algorithm.

\section{Acknowledgments}

Fund program: Scientific Research Project Plan of Shaanxi Education Department (11JK0915); Special Funding Project for High-level Universities in Shaanxi Province (Physics 2012sxts05) .

\section{References}

[1] Wu Lie, Research on Robust Optimization Design Theory and Algorithm of Electromagnetic Field Reversal, Zhejiang University, 2012;

[2] Nie Man, Research on Evolutionary Algorithms of Electromagnetic Field Reversal Analysis and Calculation, Zhejiang University, 2006;

[3] Luo Ping, Quick Global Optimization Algorithm and Its Application in High Temperature Superconducting Energy Storage Magnet, Zhejiang University, 2006;

[4] Cao Kejian, Technical Research on Robust Optimization Design of Electromagnetic Field Reversal, Zhejiang University, 2012;

[5] Yang Qingxin, An Jinlong, Ma Zhenping, Hou Likun, Chen Tanggong and Chen Haiyan, Global Optimization of Electromagnetic Field Reversal based on the Least Squares Support Vector Machine and Self-Adaption Simulated Annealing Algorithm, Transactions of China Electrotechnical Society, 2008, 11: 1-7. 